\title{
Biological predictors of survival in stage II colorectal cancer
}

\author{
YOSHITAKE UEDA ${ }^{1}$, KAZUHIRO YASUDA ${ }^{1}$, MASAFUMI INOMATA ${ }^{1}$, NORIO SHIRAISHI ${ }^{1}$, \\ SHIGEO YOKOYAMA ${ }^{2}$ and SEIGO KITANO ${ }^{3}$ \\ Departments of ${ }^{1}$ Gastroenterological Surgery and ${ }^{2}$ Pathology, Faculty of Medicine, Oita University; \\ ${ }^{3}$ Oita University, Yufu, Oita 879-5593, Japan
}

Received December 15, 2012; Accepted April 23, 2013

DOI: $10.3892 / \mathrm{mco} .2013 .126$

\begin{abstract}
The routine use of postoperative adjuvant chemotherapy in patients with stage II colorectal cancer is not recommended. However, the incidence of tumor recurrence or distant metastasis in these patients is reported to be $25-35 \%$. The identification of high-risk patients with stage II colorectal cancer remains difficult. Therefore, the aim of this study was to determine the risk factors that may help identify stage II colorectal cancer patients with unfavorable prognosis. Paraffin-embedded tissue samples from 109 patients with stage II colorectal cancer following curative operation were analyzed. Thirteen clinicopathological variables and 5 biological markers were assessed using immunohistochemistry, including p53 (tumor suppressor gene), CD10 (tumor invasion marker), CD34 (angiogenic marker), Ki-67 (cell proliferation index) and CAM 5.2 (marker of lymph node micrometastasis) and investigated for associations with disease-specific survival. Univariate analysis revealed bowel obstruction, lymph node micrometastasis and lymphatic invasion $(\mathrm{P}<0.01)$ to be highly significant factors for determining the 5-year disease-specific survival. By contrast, the multivariate analysis revealed lymph node micrometastasis and lymphatic invasion to be independent prognostic factors. Stage II colorectal cancer patients with lymph node micrometastasis and lymphatic invasion may therefore be suitable candidates for adjuvant chemotherapy to improve prognosis.
\end{abstract}

\section{Introduction}

Lymph node metastasis is the most powerful predictor of recurrence or survival in patients with colorectal cancer. Although the majority of patients with node-negative colorectal cancer are potentially cured with surgery alone, $\leq 25 \%$ are likely to present with recurrence and succumb to the disease (1).

Correspondence to: Dr Yoshitake Ueda, Department of Gastroenterological Surgery, Faculty of Medicine, Oita University 1-1 Idaigaoka, Hasama-machi, Yufu, Oita 879-5593, Japan

E-mail: yoshimd@med.oita-u.ac.jp

Key words: colorectal cancer, prognostic factor, lymphatic invasion, lymph node micrometastasis
Identifying high-risk patients with stage II colorectal cancer is important for determining which patients may benefit the most from adjuvant chemotherapy.

The effect of clinicopathological factors on recurrence and survival following curative resection has been the subject of several studies and numerous clinicopathological factors have been suggested as prognostic indicators for colorectal cancer. It is important to determine which of these factors affect the risk of recurrence in the node-negative colorectal cancer patients and which factors should be prospectively applied in the routine clinicopathological evaluation of colorectal cancer.

With the recent developments in immunohistochemistry and molecular biology, several biological markers have been extensively investigated (2-12). In this study, the expression of several biological markers, including p53, CD10, CD34 and $\mathrm{Ki}-67$, which are strongly suspected of playing a significant role in tumor progression, was evaluated. Additionally, we focused on lymph node micrometastasis, which is easily detected by antibody CAM 5.2.

The aim of this study was to conduct a multivariate analysis of the prognostic impact of a wide range of clinicopathological and biological variables in patients with stage II colorectal cancer.

\section{Materials and methods}

Patients. We reviewed all the patients who underwent curative resection for stage II colorectal cancer at the Department of Gastroenterological Surgery, Oita University Hospital, between 1984 and 2002. Patients who had received preoperative chemoradiation for locally advanced lower rectal cancer were excluded from this cohort study. Ultimately, 109 patients (61 males and 48 females; average age, 67 years; range, 31-89 years) were enrolled and the tumors were diagnosed as clinical stage T3, N0 and M0.

Evaluation. The survival analysis was performed for the following clinicopathological factors: age, gender, location of tumor (right vs. left colon vs. rectum), number of resected lymph nodes ( $0-11$ vs. $\geq 12)$, bowel obstruction (absent vs. present), tumor size (0-4 vs. $>4 \mathrm{~cm})$, depth of tumor invasion (subserosa vs. serosa), tumor differentiation (high vs. moderate vs. mucinous), lymphatic invasion (absent or mild vs. moderate or severe), venous invasion (absent vs. 
present), tumor budding (absent vs. present) (13), peritumoral lymphocytes (inconspicuous vs. conspicuous) (14) and tumor growth pattern (expansive vs. infiltrative) (14). Furthermore, 5 biological markers were asessed using immunohistochemistry, including p53 (tumor suppressor gene), CD10 (tumor invasion marker), CD34 (angiogenic marker), Ki-67 (cell proliferation index) and CAM 5.2 (marker of lymph node micrometastasis). Tumor budding is defined as an isolated single cancer cell or a cluster composed of $<5$ cancer cells observed in the stroma of the actively invasive region. A count of 0-9 per field was considered as absent and a count of $\geq 10$ was regarded as present, based on the results of a previous study (13). The characteristics of peritumoral lymphocytes (inconspicuous vs. conspicuous) and the tumor growth pattern (expansive vs. infiltrative) were assessed strictly according to the criteria originally described by Jass et al (14).

Immunohistochemistry. Resected tumors from each of the 109 patients were fixed in 10\% formalin solution and embedded in paraffin. Representative tissue sections, each containing the deepest site of cancer invasion, were cut at $4-\mu \mathrm{m}$. As regards the lymph node specimens, one $3-\mu \mathrm{m}$ section was obtained for hematoxylin and eosin staining and five serial $6-\mu \mathrm{m}$ sections for immunohistochemical staining. The avidin-biotin peroxidase complex method was used for detection of the five monoclonal antibodies in deparaffinized and rehydrated tissue sections. Antigen retrieval was performed by placing the sample in a microwave oven at $95^{\circ} \mathrm{C}$ for $40 \mathrm{~min}$, followed by cooling for $30 \mathrm{~min}$ to room temperature, except for CD34 and CAM 5.2. CAM 5.2 sections were trypsinized with $0.1 \%$ calcium chloride solution. p53, Ki-67, CD10 and CD34 were incubated for $2 \mathrm{~h}$ at room temperature and CAM 5.2 was incubated overnight at $4^{\circ} \mathrm{C}$. The slides were then incubated for 30 min with EnVision ${ }^{\mathrm{TM}}$ peroxidase mouse system (DAKO Corporation, Carpinteria, CA, USA). The color reaction product was developed with diaminobenzidine tetrahydrochloride (DAB; Sigma Chemical Co., St. Louis, MO, USA) as the chromogen for $5 \mathrm{~min}$. Using a light microscope, a visual grading system was used based on the number of positively stained nuclei of the cancer cells in each tissue sample.

p53 slides were scored according to the percentage of positive tumor nuclei as follows: positive, $\geq 10 \%$ of the nuclei stained; negative, $<10 \%$ of the nuclei stained (3). For Ki-67 immunoreactivity, staining was considered positive at $>60 \%$ (9). Tumor positivity for CD10 was evaluated using a predetermined cut-off of 5\% (positive, $>5 \%$ tumor cell staining) according to a previous study (10). CD34 slides were classified according to the microvessel count. After scanning the highly vascularized areas, we selected three areas exhibiting the most prominent neovascularization. A microvessel count was performed on a $\times 400$ field (x40 objective and $x 10$ ocular) and the average count from the three areas was calculated (15). Patients were divided into those with a microvessel count of 0-50 and those with a microvessel count of $>50$. As regards metastatic lymph nodes, patients were divided into two groups according to a previous study (16), those with micrometastasis in $\leq 3$ lymph nodes and those with micrometastasis in $\geq 4$ lymph nodes. Written informed consent was obtained from all the patients and this study was approved by the local Ethics Committee.

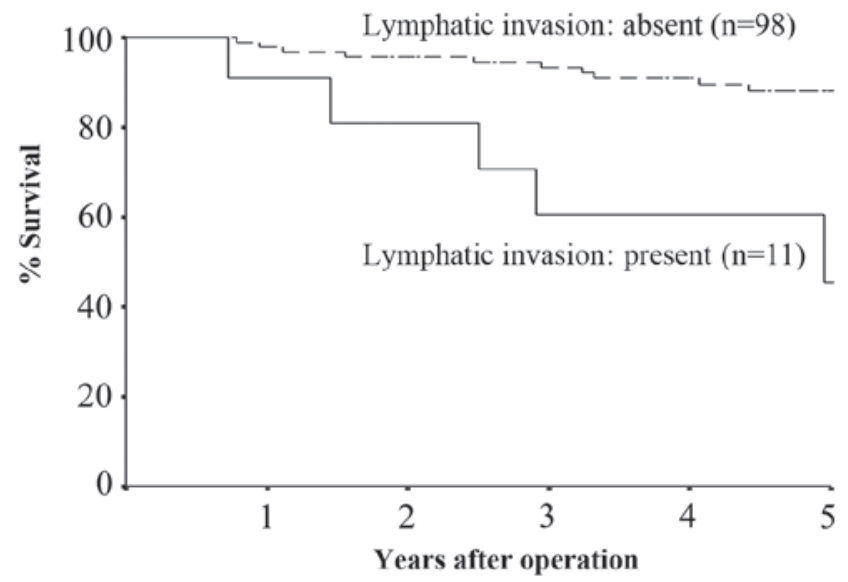

Figure 1. The 5-year disease-specific survival rate for the group of patients with lymphatic invasion was significantly lower compared to that for the group without lymphatic invasion ( 55 vs. $90 \%, \mathrm{P}<0.01)$.

Statistical analysis. Data were statistically analyzed using SPSS statistical software (Statistical Package for Social Sciences). Univariate disease-specific survival analysis was performed using the Kaplan-Meier method and the difference was evaluated by the log-rank test. Multivariate analysis was performed using the Cox proportional hazards model. $\mathrm{P}<0.05$ was considered to indicate a statistically significant difference.

\section{Results}

Factors affecting patient survival. The study included a total of 61 males and 48 females, with an average age of 67 years (range, 31-89 years). The median follow-up period for the survivors was 5.7 years (range, 1.7-11 years). At the time of analysis, 87 patients were free of disease, 7 were alive with disease, 15 had succumbed to the disease and 5 patients had succumbed due to other causes. Twenty-two patients developed recurrence or distant metastasis. Of these, 12 had liver metastases, 7 had local recurrence and 3 had lung metastasis. The 5 -year disease-specific survival rate of patients with stage II colorectal cancer was $86.2 \%$.

In the univariate analysis, bowel obstruction, lymph node micrometastasis and lymphatic invasion $(\mathrm{P}<0.01)$ were significant factors for determining the 5-year disease-specific survival (Table I). When all of these factors were included as independent variables in a Cox proportional hazards model, the presence of lymphatic invasion was the most powerful negative predictor of survival [hazard ratio (HR), 4.091; $\mathrm{P}=0.006]$, followed by lymph node micrometastasis (HR, 3.704; $\mathrm{P}=0.011$ ) (Table II). The 5-year disease-specific survival rate was significantly lower for the group of patients with moderate to severe presence of lymphatic invasion compared to that for the group with absent to mild presence of lymphatic invasion (55 vs. $90 \%, \mathrm{P}<0.01$ ) (Fig. 1). Similarly, the 5-year disease-specific survival rate was significantly lower for the group with $\geq 4$ positive micrometastatic nodes compared to that for the group with $0-3$ positive micrometastatic nodes (46 vs. $92 \%, \mathrm{P}<0.01$ ) (Fig. 2). When a combination of two factors, lymphatic invasion and micrometastasis was examined, the 5-year disease-specific survival rate for the group of 
Table I. Univariate analysis for the 5-year disease-specific survival in patients with stage II colorectal cancer.

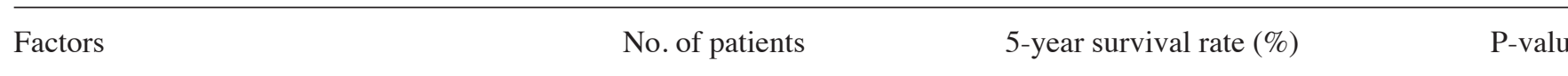

Age (years)

$0-70$

$\geq 71$

Gender

Male

Female

Location of tumor

Right colon

No. of resected lymph nodes

0-11

$\geq 12$

Bowel obstruction

Absent

Present

Tumor size $(\mathrm{cm})$

$0-4$

$>4$

Depth of tumor invasion

T3

Differentiation

High

Moderate

Lymphatic invasion

Absent, mild

Moderate, severe

Venous invasion

Absent

Present

Tumor budding

Absent

Present

Peritumoral lymphocytes

Inconspicuous

Tumor growth pattern

Expansive

Infiltrative

p53

Negative

Positive

CD10
$<0.01$ 
Table I. Continued.

\begin{tabular}{|c|c|c|c|}
\hline Factors & No. of patients & 5-year survival rate $(\%)$ & P-value \\
\hline \multicolumn{4}{|c|}{ Angiogenesis (microvessel count) } \\
\hline $0-50$ & 90 & 80 & NS \\
\hline$>50$ & 19 & 79 & \\
\hline \multicolumn{4}{|l|}{ Ki-67 index } \\
\hline Sparse & 94 & 78 & NS \\
\hline Diffuse & 15 & 93 & \\
\hline \multicolumn{4}{|c|}{ Lymph node micrometastasis } \\
\hline 0-3 positive nodes & 96 & 92 & $<0.01$ \\
\hline$\geq 4$ positive nodes & 13 & 46 & \\
\hline
\end{tabular}

NS, non-significant.

Table II. Multivariate analysis for the 5-year disease-specific survival in patients with stage II colorectal cancer.

\begin{tabular}{lccc}
\hline Factors & HR & $95 \%$ CI & P-value \\
\hline Lymphatic invasion & 4.091 & $1.376-12.165$ & 0.006 \\
Lymph node micrometastasis & 3.704 & $1.458-9.406$ & 0.011 \\
\hline
\end{tabular}

HR, hazard ratio; CI, confidence interval.

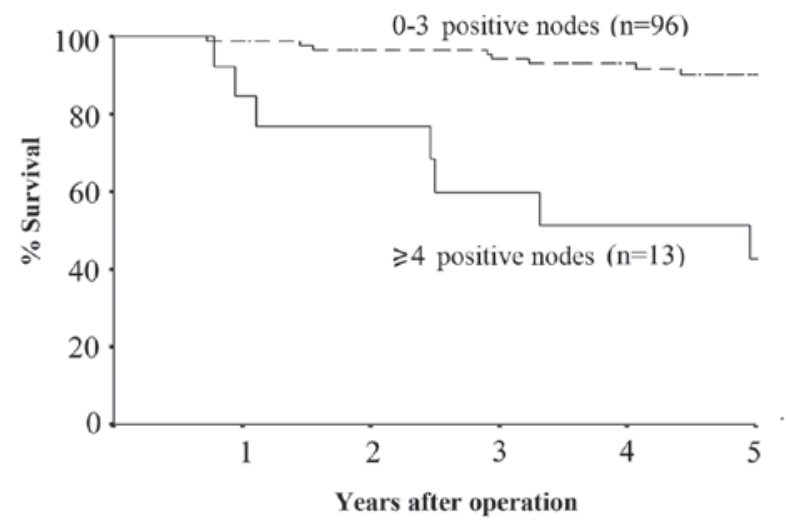

Figure 2. The 5-year disease-specific survival rate for the group of patients with $\geq 4$ micrometastases was significantly lower compared to that for the group with $0-3$ micrometastases (46 vs. $92 \%, \mathrm{P}<0.01$ ).

patients with either one positive factor was significantly lower compared to that for the group with both factors negative (55 vs. 94\%, $\mathrm{P}<0.01)$ (Fig. 3).

\section{Discussion}

In the present study, $20 \%$ of the stage II colorectal cancer patients presented with tumor recurrence or distant metastasis during follow-up, after curative resection. A multivariate analysis allowed us to define a subgroup of patients at high risk of recurrence, which included those with lymph node micrometastasis and those with lymphatic invasion. In addition,

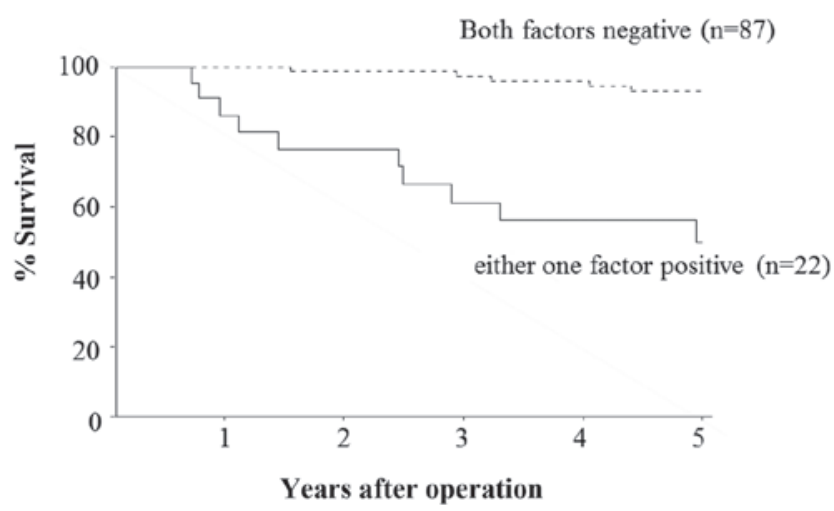

Figure 3. Lymphatic invasion and micrometastasis: the 5-year disease-specific survival rate for the group of patients with either one positive factor was significantly lower compared to that for the group with both factors negative (55 vs. $94 \%, \mathrm{P}<0.01)$.

these factors were significantly associated with the prognosis of stage II colorectal cancer patients.

Recent advances in immunohistochemistry and molecular biology suggest that molecular changes of the primary tumor may serve as prognostic indicators for individual patients. Several studies have attempted to identify the prognostic biomarkers in patients with stage II or node-negative colorectal cancer $(2-7,12,17,18)$.

Although several studies have been conducted on lymph node micrometastasis in patients with colorectal cancer, the significance of the presence of lymph node micrometastasis has been a subject of debate (16,18-21). Yasuda et al (16) 
reported that micrometastasis in $\geq 4$ lymph nodes and micrometastasis to $\mathrm{N} 2$ or higher nodes were significantly correlated with postoperative recurrence and prognosis in stage II colorectal cancer patients. Bukholm et al (21) reported that the presence of isolated tumor cells in the mesenteric lymph nodes was independently associated with reduced relative survival in patients with stage II colon cancer. Our study also demonstrated that the number of lymph node micrometastases was a more powerful indicator than the presence and level of lymph node micrometastasis. Therefore, it is helpful to investigate the number of lymph node micrometastases with immunohistochemistry in stage II colorectal cancer patients.

The aim of adjuvant chemotherapy is the destruction of microscopic metastases that may already be present and the reduction of the risk of recurrence. Postoperative chemotherapy for stage III colorectal cancer patients has been shown to improve prognosis and is recommended as standard therapy $(22,23)$. However, the value of adjuvant chemotherapy for patients with stage II colorectal cancer is controversial $(24,25)$. The International Multicentre Pooled Analysis of B2 Cancer Trials (IMPACT B2) (26) and the meta-analysis reported by Figueredo et al (27) did not demonstrate any improvement in prognosis of stage II colon cancer patients treated with adjuvant chemotherapy. However, the QUASAR study demonstrated a significantly reduced recurrence rate and improved survival of patients with stage II colorectal cancer in favour of the adjuvant chemotherapy arm (28).

Although several large studies have investigated the subject of adjuvant chemotherapy for stage II colorectal cancer patients, the use of adjuvant chemotherapy for all stage II colorectal cancer patients may be inappropriate and expensive (29). Therefore, there is an increasing need for accurate stratification of stage II colorectal cancer patients in order to identify those at high-risk of recurrence who may benefit from adjuvant chemotherapy.

Our data suggest that two factors, lymph node micrometastasis and lymphatic invasion, should be included in the high-risk group of patients with stage II colorectal cancer. Sirop et al (30) reported improved outcomes of micrometastasis after being considered as high-risk disease and treated with chemotherapy in their pilot study. These results suggest a trend in favour of adjuvant chemotherapy in stage II colorectal cancer patients with high-risk factors.

In conclusion, we demonstrated that each of the two factors investigated, lymph node micrometastasis and lymphatic invasion, carries independent prognostic significance with respect to the 5-year disease-specific survival rates of patients with stage II colorectal cancer. This finding may be useful in identifying the high-risk patients for recurrence or metastasis among stage II colorectal cancer patients. We recommend that stage II colorectal cancer patients with lymph node micrometastasis and lymphatic invasion be evaluated for the benefit of adjuvant chemotherapy in the future, through further prospective randomized control studies.

\section{Acknowledgements}

This study was supported in part by the National Cancer Center Research and Development Fund (23-A-19).

\section{References}

1. Jemal A, Siegel R, Ward EA, et al: Cancer statistics, 2009. CA Cancer J Clin 59: 225-249, 2009.

2. Graziano F and Cascinu S: Prognostic molecular markers for planning adjuvant chemotherapy trials in Dukes' B colorectal cancer patients: how much evidence is enough? Ann Oncol 14: 1026-1038, 2003

3. Allegra CJ, Paik S, Colangelo LH, et al: Prognostic value of thymidylate synthase, $\mathrm{Ki}-67$, and p53 in patients with Dukes' B and C colon cancer: a National Cancer Institute-National Surgical Adjuvant Breast and Bowel Project collaborative study. J Clin Oncol 21: 241-250, 2003.

4. Garrity MM, Burgart LJ, Mahoney MR, et al: Prognostic value of proliferation, apoptosis, defective DNA mismatch repair, and p53 overexpression in patients with resected Dukes' B2 or C colon cancer: a North Central Cancer Treatment Group Study. J Clin Oncol 22: 1572-1582, 2004.

5. Bhatavdekar JM, Patel DD, Chikhlikar PR, et al: Molecular markers are predictors of recurrence and survival in patients with Dukes B and Dukes C colorectal adenocarcinoma. Dis Colon Rectum 44: 523-533, 2001.

6. Gervaz P, Cerottini JP, Bouzourene $\mathrm{H}$, et al: Comparison of microsatellite instability and chromosomal instability in predicting survival of patients with T3N0 colorectal cancer. Surgery 131: 190-197, 2002.

7. Sinicrope FA, Hart J, Hsu HA, et al: Apoptotic and mitotic indices predict survival rates in lymph node-negative colon carcinomas. Clin Cancer Res 5: 1793-1804, 1999.

8. Kim YH, Lee JH, Chun H, et al: Apoptosis and its correlation with proliferative activity in rectal cancer. J Surg Oncol 79: 236-242, 2002.

9. Sadahiro S, Suzuki T, Maeda Y, et al: Predictors of tumor downsizing and regression with preoperative radiotherapy alone and with concomitant tegafur/uracil (UFT) for resectable advanced rectal adenocarcinoma. Hepatogastroenterology 54: 1107-1112, 2007.

10. Fujimoto Y, Nakanishi Y, Sekine S, et al: CD10 expression in colorectal carcinoma correlates with liver metastasis. Dis Colon Rectum 48: 1883-1889, 2005.

11. Ogawa H, Iwaya K, Izumi M, et al: Expression of CD10 by stromal cells during colorectal tumor development. Hum Pathol 33: 806-811, 2002.

12. Weber JC, Nakano H, Bachellier P, et al: Is a proliferation index of cancer cells a reliable prognostic factor after hepatectomy in patients with colorectal liver metastases? Am J Surg 182: 81-88, 2001.

13. Ueno $\mathrm{H}$, Price $\mathrm{AB}$, Wilkinson $\mathrm{KH}$, et al: A new prognostic staging system for rectal cancer. Ann Surg 240: 832-839, 2004.

14. Jass JR, Love SB and Northover JM: A new prognostic classification of rectal cancer. Lancet 1: 1303-1306, 1987.

15. Oh-e H, Tanaka S, Kitadai Y, et al: Angiogenesis at the site of deepest penetration predicts lymph node metastasis of submucosal colorectal cancer. Dis Colon Rectum 44: 1129-1136, 2001.

16. Yasuda K, Adachi Y, Shiraishi N, et al: Pattern of lymph node micrometastasis and prognosis of patients with colorectal cancer. Ann Surg Oncol 8: 300-304, 2001.

17. Okuyama T, Nakamura T and Yamaguchi M: Budding is useful to select high-risk patients in stage II well-differentiated or moderately differentiated colon adenocarcinoma. Dis Colon Rectum 46: 1400-1406, 2003.

18. Feezor RJ, Copeland EM III and Hochwald SN: Significance of micrometastases in colorectal cancer. Ann Surg Oncol 9: 944-953, 2002.

19. Palmqvist R, Sellberg P, Oberg A, et al: Low tumour cell proliferation at the invasive margin is associated with a poor prognosis in Dukes' stage B colorectal cancers. Br J Cancer 79: 577-581, 1999.

20. Noura S, Yamamoto H, Miyake Y, et al: Immunohistochemical assessment of localization and frequency of micrometastases in lymph nodes of colorectal cancer. Clin Cancer Res 8: 759-767, 2002.

21. Bukholm IR, Bondi J, Wiik P, et al: Presence of isolated tumour cells in mesenteric lymph nodes predicts poor prognosis in patients with stage II colon cancer. Eur J Surg Oncol 29: 862-866, 2003.

22. O'Connell MJ, Mailliard JA, Kahn MJ, et al: Controlled trial of fluorouracil and low-dose leucovorin given 6 months as postoperative adjuvant therapy for colon cancer. J Clin Oncol 15: 246-250, 1997. 
23. Barone C: Adjuvant chemotherapy of colon cancer current strategies. Eur J Cancer 6: 60-63, 2008.

24. Van Cutsem E and Costa F: Progress in the adjuvant treatment of colon cancer. Has it influenced clinical practice? JAMA 7: 2758-2760, 2005.

25. O'Connell MJ: Oxaliplatin or irinotecan as adjuvant therapy for colon cancer: the results are in. J Clin Oncol 27:3082-3084, 2009.

26. No authors listed: Efficacy of adjuvant fluorouracil and folinic acid in B2 colon cancer. International Multicentre Pooled Analysis of B2 Colon Cancer Trials (IMPACT B2) Investigators. J Clin Oncol 17: 1356-1363, 1999.

27. Figueredo A, Charrette M, Maroun J, et al: Adjuvant therapy for stage II colon cancer: a systematic review from the Cancer Care Ontario Program in evidence-based care's gastrointestinal cancer disease site group. J Clin Oncol 22: 3395-3407, 2004.
28. Quasar Collaborative Group, Gray R, Barnwell J, McConkey C, Hills RK, Williams NS and Kerr DJ: Adjuvant chemotherapy versus observation in patients with colorectal cancer: a randomised study. Lancet 370: 2020-2029, 2007.

29. Norum J: Adjuvant chemotherapy in Dukes' B and C colorectal cancer has only a minor influence on psychological distress. Support Care Cancer 5: 318-321, 1997.

30. Sirop S, Kanaan M, Korant A, et al: Detection and prognostic impact of micrometastasis in colorectal cancer. J Surg Oncol 103: 534-537, 2011. 